\title{
Estilos de aprendizaje $y$ rendimiento académico en estudiantes del primer año de Medicina Humana (2016- 2017) en la Universidad Nacional Mayor de San Marcos
}

\author{
First year medical students' learning styles and performance (2016-2017) at Universidad Nacional \\ Mayor de San Marcos \\ Mirko Humberto Hoyos-Flores ${ }^{1}$, Hallder Mori-Ramírez ${ }^{2}$
}

\section{RESUMEN}

Introducción: Los estilos de aprendizaje son estudiados por su creciente utilidad en la formulación de estrategias de enseñanza acordes al perfil de cada estudiante. El rendimiento académico es la medida más fiable usada para comprobar la eficacia del método de estudio empleado, actualmente no se conoce la relación entre estilos y rendimiento académico en la Escuela de Medicina de la Universidad Nacional Mayor de San Marcos. Objetivo: El estudio tuvo como meta principal la identificación de los estilos de aprendizaje utilizados por los estudiantes de medicina en el primer año, y su relación con el rendimiento académico. Materiales y Métodos: La muestra consistió de 234 estudiantes de primer año durante el periodo 2016 y 2017. Se aplicó el cuestionario Honey-Alonso de estilos de aprendizaje, clasificándolos como activo, reflexivo, teórico o pragmático. Se relacionó el nivel de las preferencias en los estilos únicos y se buscó la relación con los rendimientos académicos basados en el promedio ponderado. Resultados: La edad promedio de los participantes fue de 19 años. El estilo predominante fue el reflexivo (70.19\%), luego el teórico (13.94\%), pragmático (10.58\%) y el activo (5,29\%). Se evidenció el dominio de dos estilos en 22 estudiantes y tres estilos en 3 estudiantes. No se encontró diferencias significativas en los rendimientos de grupos con diferentes estilos de aprendizaje. Conclusiones: El estilo predominante fue el reflexivo, con una preferencia mediana. No existe una relación estadísticamente significativa entre el rendimiento académico y los estilos de aprendizaje.

Palabras clave: estilos de aprendizaje, rendimiento académico, cuestionario Honey-Alonso.

\begin{abstract}
Introduction: The learning styles were studied decades ago because of their usefulness for the creation of strategies following the students' profile, in different education levels and courses. The performance achieved is considered in order to evaluate the efficiency of the strategies. So far, there is not a study that relates the learning style with the academic performance. Objective: This study has its goal in the identification of the learning styles used by the first year medical students, according to their preference level, and the relation between the style and the academic performance. Materials and Methods: The sample consisted of 234 students that were taking the first year on 2016 and 2017. We applied the Honey-Alonso questionary in order to classify the participants by their learning styles as active, reflective, theoretical, and pragmatic. We calculated also the preference of each style and searched for the relation between them and the academic performance. Results: The participants were 19 years old in average. The predominant style was the reflective (70.19\%), following the theoretical (13.94\%), pragmatic (10.58\%) and the active (5.29\%). We evidenced the domain of two styles among 22 students and three styles in 3 students and we didn't find any statistical relation between the styles and the performance. Conclusion: The reflective prevailed and had a moderate preference, the other styles had a very high preference. There is not relation between the learning styles and the academic performance.
\end{abstract}

Keywords: Learning styles, academic performance, Honey-Alonso cuestionary.

${ }^{1}$ Unidad de Posgrado. Facultad de Medicina "San Fernando". Universidad Nacional Mayor de San Marcos

${ }^{2}$ Profesor Principal TC. Facultad de Medicina. Universidad Nacional Mayor de San Marcos. Lima - Perú. 


\section{INTRODUCCIÓN}

Los estilos de aprendizaje se conceptúan como características de índole cognitivas y emocionales vinculadas a particularidades biológicas de los estudiantes que están en constante aprendizaje de datos (1).

Las investigaciones sobre el estilo de aprendizaje aportaron valiosa información a los procesos educativos. En el año 1976, Kolb planteó a las características psicológicas y destrezas de acuerdo a la especialidad relacionada con las labores como influencias importantes en el aprendizaje; en 1986, Honey y Mumford elaboraron un cuestionario que determina 4 estilos de aprendizaje: Reflexivo, Activo, Teórico y Pragmático; años después, Alonso lo adaptó al entorno estudiantil, denominándolo Cuestionario Honey-Alonso sobre Estilos de Aprendizaje o CHAEA; siendo criterios que definen al cuestionario que identifica estilos de aprendizaje:

- La importancia teórica en el campo como un todo,

- el uso generalizado, ya sea en el mercado o académicamente y

- la influencia en otros modelos/instrumentos de estilos de aprendizaje $(2,13)$.

\section{INTERPRETACIÓN.-}

- Si más de la mitad de preguntas concernientes a un estilo se responden de manera positiva, entonces se puede considerar a ese estilo como propio del perfil,

- si tiene menos del 50\% de respuestas positivas, se considerará como estilo adicional para aprender $(2,6)$.

Las características que definen a cada estilo son (3):

- Activo: Buscan algo novedoso tanto en experiencias como retos.

- Reflexivo: Prefieren tomar un tiempo analizando desde diferentes puntos de vista antes de concluir una afirmación, son prudentes.

- Teórico: Buscan el mayor grado de efectividad y de eficiencia. Integran teorías acordes con los hechos basados en raciocinios y juicios objetivos.

- Pragmático: Se esmeran por relacionar una afirmación con su aplicación en la realidad, sus conclusiones sobre un tema se basan en la utilidad.
Cala, Riera y Jaramillo (2014) elaboraron un estudio en estudiantes de ingeniería en el que se observó preferencia similar por los estilos activo y teórico, siendo los estilos predominantes el pragmático y el activo; en ingeniería industrial tuvieron una menor tendencia a lo reflexivo y más a lo pragmático que en ingeniería electrónica. Esta base sentó un precedente para los desarrollos de métodos de enseñanza personalizada (3).

El promedio ponderado es usado como predictor del rendimiento académico en gran cantidad de estudios (5).

Torres y Díaz, (2016) publicaron un trabajo de investigación que buscó relacionar los estilos de aprendizaje y el rendimiento académico de alumnos de Medicina Humana del primero al sexto año en la Universidad San Martin de Porres en Perú, se encontró una mayoría que usa el estilo reflexivo siguiendo en orden decreciente a los que usaban los estilos teórico, pragmático y finalmente activo. Se observó también una relación significativa solo entre el estilo teórico y el rendimiento académico (4).

Caballero y colaboradores estudiaron al rendimiento académico en relación a los estilos de aprendizaje en estudiantes de Trabajo Social en Chile y México, en el primer país el estilo predominante fue el reflexivo mientras que en el segundo fue el teórico en combinación con el pragmático. En ambos casos los resultados coincidieron con estudios anteriores realizados en los mismos territorios; los estudios mostraron un nivel de rendimiento académico "regular", lo que sugiere que, al estilo adoptado, se agregan otros factores que influyen en el rendimiento académico (2).

García, Tamez y Lozano concluyeron en su estudio realizado en estudiantes de secundaria el año 2015 en Colombia que un estilo de aprendizaje no es mejor que otro, al observar que alumnos con altas calificaciones en los cursos de español y matemáticas se encuentra una preferencia moderada, mencionaron que cada estilo tiene un valor adicional y útil ante situaciones particulares (5). El rendimiento en su mayoría se compone de la recopilación directa de calificaciones, en estudios anteriores se usó como una variable que describe efectividad en estrategias didácticas.

Alvarado, (2017) en Colombia observó falta de conocimiento en los docentes acerca de los estilos de aprendizaje, por lo que en base a éstos se recomendó el diseño de estrategias de enseñanza-aprendizaje (7), entendiéndolas como un grupo de procesos usados convenientemente por el alumno para adaptarse a la asimilación de conocimientos (1). 
El estudio realizado por Alzate en el año 2014 señaló que los estudiantes con un rango de edad entre los 12 y 18 años usan el estilo activo $(8,9)$, mientras que el estudio de Ángel y Alonso, hecho en el año 2012 refirió, que a medida que la edad avance, las personas marcarían una mayor tendencia a ser del estilo teórico (que busca efectividad y eficiencia), los jóvenes serían más activos (8).

El aprendizaje debería seguir un ciclo que empieza con una intención y estrategias para la búsqueda de datos (estilo activo), para luego discutir sobre la información recopilada (estilo reflexivo), entonces armar un esquema concreto a modo de teorema (estilo teórico) y al final practicar la teoría en situaciones reales (estilo pragmático); el ciclo se repetiría a partir de ese punto. Martínez e investigadores asociados demostraron en su investigación la prevalencia del estilo reflexivo por encima de los demás estilos, aunque con una preferencia moderada; sin embargo, el estilo activo tuvo una muy baja preferencia, comprobando un defecto en el ciclo de aprendizaje; también señalaron que el rendimiento académico más alto se dió en quienes mostraron predominio de más de un estilo (10).

En el estudio de Estrada y Alejandro se halló la prevalencia de los estilos de aprendizaje Activo y Teórico en estudiantes de Química, con una preferencia moderada; por otro lado, los estilos pragmático y reflexivo tuvieron una calificación de preferencia menor, la no correspondencia con el perfil de ingreso hizo sugerir la implementación de métodos por parte de la comunidad docente para incrementar estilos de aprendizaje acordes a las habilidades esperadas de los estudiantes (11), se debe considerar la posibilidad de identificar el estilo al egreso de la carrera (6), teniendo en cuenta que será el que guiará su comportamiento como profesional.

El estudio de Díaz y Ortega buscó indagar las diferencias en los estilos en grupos de turnos diferentes en tres carreras de ingeniería (jornada diurna y jornada vespertina). Los resultados no presentaron diferencias significativas entre grupos de ambas jornadas, lo que llevó a los autores a sugerir un estudio en jornada vespertina, ya que se conforma por alumnos de mayor edad y pasando a la etapa adulta (12).

En su estudio, Aravena y colaboradores no encontraron diferencia entre los estilos, relacionándolos con el coeficiente intelectual (14). El estilo elegido por el alumno no es inherente a la profesión estudiada, pero se influye por rasgos de personalidad, y no es invariable, tampoco excluye a los demás estilos de aprendizaje y su uso dependerá también de las experiencias vividas (15). Los estilos de aprendizaje comprenden el apoyo en diferentes sectores del conocimiento tales como la didáctica, pedagogía y psicología gracias a trabajos de investigación anteriores. A la actualidad, sirve como guía para el profesor, pues puede conocer las tendencias de preferencia para cada uno de los estilos y así proporcionar una educación individualizada. $(7,13)$.

En el presente estudio se tuvo como objetivo determinar los estilos de aprendizaje utilizados por los estudiantes de medicina en el primer año en la Universidad Nacional Mayor de San Marcos, así como el grado de preferencia, y su relación con el rendimiento académico.

\section{MATERIALES Y MÉTODOS}

\section{Diseño de estudio}

Estudio analítico, correlacional, observacional y transversal con enfoque cuantitativo y diseño no experimental. El presente estudio se realizó en las instalaciones de la Facultad de Medicina Humana de "San Fernando" en el periodo comprendido entre los años 2016 y 2017.

\section{Población y Muestra}

La población objeto de estudio estuvo conformada por todos los estudiantes de primer año de la Escuela Profesional de Medicina Humana de la Universidad Nacional Mayor de San Marcos. Se seleccionaron a los participantes de las promociones ingresantes en los años 2016 y 2017 , que aceptaron participar en el estudio y se encontraban en situación regular (llevaban la lista completa de cursos), equivalente a 234 estudiantes.

\section{Variables}

- Estilos de Aprendizaje: Habilidades para aprender que sobresalen debido a la interacción entre experiencias y las demandas en el entorno del estudiante (5).

- Rendimiento Académico: Característica cambiante constantemente, conformada por indicadores objetivos (como las calificaciones) y otros que pueden ser acoplados según la materia (5).

\section{Procedimiento}

Se utilizó el Cuestionario Honey-Alonso de Estilos de Aprendizaje (CHAEA). Dicho formato consta con un total de 80 enunciados que se acompañan con un casillero que será marcado en caso de respuesta positiva, de los cuales 20 corresponden a cada estilo: Activo, reflexivo, 
teórico y pragmático. Dicho instrumento fue sometido a pruebas de fiabilidad con respecto a cada estilo en el año 1997 por Alonso con la prueba Alfa de Cronbach, a su vez el cuestionario fue analizado por 16 jueces. Posteriormente fue validado y adaptado en Perú por Capella y colaboradores en el año 2002 con la misma prueba de Alfa de Cronbach. Alonso y colaboradores en el año 2012, indicaron coeficientes de confiabilidad entre 0,58 y 0,72 . Siendo que los valores aceptados para el coeficiente Alfa de Cronbach se dan a partir de 0,6 (13).

Para el rendimiento académico se usó la recopilación directa de los promedios ponderados anuales otorgados por la universidad.

\section{Aspectos éticos}

Se explicó a los participantes acerca de los objetivos de la encuesta, así como el carácter voluntario de la participación y los beneficios de conocer el estilo de aprendizaje que se posee.

Los autores del estudio pidieron permiso a la Dirección de la Escuela de Medicina comprometiéndose a usar los datos con el propósito exclusivo de aplicarlos en el presente estudio; a su vez, se le proporcionó a cada participante un código a fin de no usar sus datos personales posteriormente al procesado de datos.

\section{Análisis de datos}

Los datos recopilados fueron plasmados en una plantilla Excel 2013. La preferencia del aprendizaje se calculó desde los puntajes que cada estudiante definió en el cuestionario para cada estilo y teniendo en cuenta los niveles ordinales (baja de 0 a 11, mediana de 12 a 18 y alta prevalencia de 19 a 20) correspondientes a Rho Spearman, el cual fue usado en la estadística inferencial.

\section{RESULTADOS}

La edad media encontrada resultó ser 19 años, con una desviación estándar de 1,964. La edad mínima encontrada fue de 16 años y la máxima de 30 años.

La prevalencia para los estilos fue considerada por separado en los grupos con un solo estilo ponderante con respecto a aquellos con dos o más estilos de igual dominancia. Los participantes con un estilo de aprendizaje comprendieron un $88.89 \%$ del total, mientras que los que usan dos estilos al mismo tiempo juntos fueron $9.83 \%$ y los que usan 3 estilos a la vez llegaron a 1.28\% (Tabla 1).
Tabla 1. Distribución de la muestra para los estilos de aprendizaje empleados.

\begin{tabular}{|c|c|c|c|}
\hline Estilo & Muestra & Porcentaje & Total \\
\hline TOSTEO & 70 & 12.7946 & \multirow{4}{*}{80.004} \\
\hline Aetvo & 11 & $47 \mathrm{nth}$ & \\
\hline Aeflesovo & 146 & 82.995 & \\
\hline Pragmatioo & 22 & \# $40 \%$ & \\
\hline Rentenvo y Teonco & 18 & Eबा\% & \multirow{5}{*}{ encos } \\
\hline Reflexwo y Pragmatico & 3 & $1.218 \%$ & \\
\hline Activo y Pragmatico. & 1 & $0.43 \%$ & \\
\hline Antwo y Reflewvo & 2 & $0.05 \%$ & \\
\hline Terico y Aragmaton & 2 & a. ans & \\
\hline 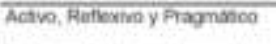 & 2 & 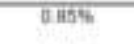 & \multirow{2}{*}{$128 \%$} \\
\hline Reflesivo Teonco y Pragmatico & 1 & 0.435 & \\
\hline TOTAL & 234 & $100.00 \%$ & $100.00 \%$ \\
\hline
\end{tabular}

Fuente: Autores, Excel 2013.

Como se observa en la Figura 1; dentro de los que siguen un sólo estilo, el estilo de aprendizaje predominante es el reflexivo con un $70.19 \%$, seguido del teórico con $13.94 \%$, el pragmático con un $10.58 \%$ y el activo con un $5.29 \%$. En la misma Figura 1; dentro de la práctica de dos estilos de aprendizaje por los estudiantes de medicina, la combinación predominante fue reflexivo-teórico con un $65.22 \%$; seguida de reflexivo-pragmático con un $13.04 \%$, teórico-pragmático y el Activoreflexivo $(8.70 \%)$ y por último Activo-Pragmático con un $4.35 \%$. Dentro de la práctica de tres estilos de aprendizaje, la combinación prevalente fue activo, reflexivo y pragmático con un $66.67 \%$; seguido de reflexivo, teórico y pragmático con un 33.33 .
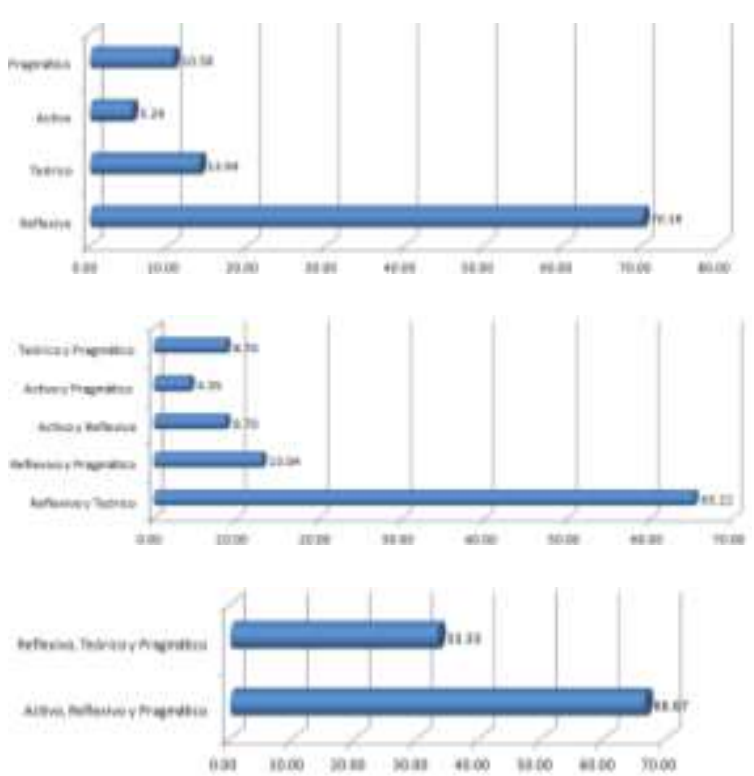

Figura 1. Distribución de porcentajes hallados en participantes con un estilo, también dos y tres estilos de igual dominancia (Fuente: Autores, Excel 2013). 
En cuanto a las preferencias, la mayoría de estudiantes ubicaron tendencias a un mediano grado en los estilos reflexivo y teórico, situando en cada grupo a la minoría entre las alta y baja preferencias. En los grupos con ponderancia de los estilos activo y pragmático, la mediana preferencia quedó en primer lugar.

Como se puede observar en la Tabla y Figura anteriores, las notas en los diversos estilos oscilan entre 13.21 y 14.80 lo que implica una diferencia de 13 a 15, sin embargo, si se ajusta a una muestra más significativa considerando cantidad de estudiantes entre 11 y 146 las notas promedios varían descriptivamente entre 13.38 y 14.34 , lo que redondeando implica una variación de 13 a 14 , lo cual no es un diferencia relevante. Por tanto, descriptivamente todos los estilos presentan promedios de notas similares.

Tabla 2. Clasificación de la preferencia para los 4 estilos de aprendizaje.

\begin{tabular}{|c|c|c|c|}
\hline Estile Reflexivo & Frecuencia & Poreentaje & $\begin{array}{l}\text { Purcentage } \\
\text { acumalado }\end{array}$ \\
\hline Bust Preferencas & $\frac{2}{2}$ & 1.4 & 1.4 \\
\hline $\begin{array}{l}\text { Medauna } \\
\text { Preferencia }\end{array}$ & 117 & 80,1 & 815 \\
\hline Ata Prefertacia & 27 & 18.5 & 100,0 \\
\hline Toal & 146 & 100.0 & \\
\hline Estile Teórice & Frecuencia & Porcentaje & $\begin{array}{l}\text { Porcentage } \\
\text { acunualado }\end{array}$ \\
\hline Baja Preferencia & 3 & 103 & 10,3 \\
\hline $\begin{array}{l}\text { Medanza } \\
\text { Preferencia }\end{array}$ & 15 & 51,7 & 62,1 \\
\hline Alta Preferencis & 11 & 37,9 & 100,0 \\
\hline Toenl & 29 & 100.0 & \\
\hline Estilo Activo & Frecuencia & Potcentage & $\begin{array}{l}\text { Porcentaje } \\
\text { acumulado }\end{array}$ \\
\hline Baja Preferencia & 3 & 273 & 27,3 \\
\hline $\begin{array}{l}\text { Medana } \\
\text { Preferencia }\end{array}$ & 5 & 45,5 & 72,7 \\
\hline Alta Prefertacis & 3 & 273 & 100,0 \\
\hline Toesl & 11 & 1000 & \\
\hline Estile Praganatico & Frecuencia & Porcentaje & $\begin{array}{l}\text { Porcentave } \\
\text { acumulado }\end{array}$ \\
\hline Baga Preferencia & 3 & 13,6 & 13,6 \\
\hline $\begin{array}{l}\text { Mediana } \\
\text { Preferencia }\end{array}$ & 10 & 45,5 & $\$ 9,1$ \\
\hline Alta Prefertacia & 9 & 40,9 & 100,0 \\
\hline Tocal & 22 & 100,0 & \\
\hline
\end{tabular}

Fuente: Autores, Excel 2013.

Tabla 3. Clasificación de la preferencia para los 4 estilos de aprendizaje.

\begin{tabular}{|c|c|c|}
\hline Estile & Muestra & Nota Promedio \\
\hline Teórico & 29 & 14.14 \\
\hline Activo & 11 & 1338 \\
\hline Reflexive & 146 & 1433 \\
\hline Pragmátice & 22 & 13.93 \\
\hline Reflexive y Teórico & 15 & 1434 \\
\hline Reflexivo y Pragmático & 3 & 13,70 \\
\hline Actvo y Pragmatico & 1 & 14.80 \\
\hline Activo y Rellexivo & 2 & 13.87 \\
\hline Teórico y Prazmàtico & 2 & 13,41 \\
\hline Activo, Reflexivo y Pragmätico & 2 & 1432 \\
\hline Reflexive, Tebrice y Pragmatice & 1 & 13.21 \\
\hline
\end{tabular}

Fuente: Autores, Excel 2013.
La prueba Rho de Spearman mostró que en ninguno de los estilos existe una relación significativa $(p>0,05)$ y que particularmente en los grupos con estilos teórico, activo y pragmático; se da una relación inversa al tener coeficientes de correlación negativos (ver Tabla 4).

En relación a la preferencia de cada estilo y relacionándola con el rendimiento académico, en el grupo con estilo reflexivo podemos observar que las calificaciones más altas están en aquellos que tienen una alta preferencia. Mientras que en el grupo con demás estilos ponderantes, las calificaciones más altas se tuvieron en quienes tenían bajas preferencias.

\section{DISCUSIÓN}

La prevalencia hallada con respecto a estilos de aprendizaje es congruente con la modalidad de aprendizaje analítica del ingresante a la Escuela de Medicina Humana de la Universidad Nacional Mayor de San Marcos, es necesario recalcar el carácter no excluyente de los estilos y su posible cambio en el estudiante, en la medida que los estudios, por su naturaleza, les exijan adoptar otros estilos de aprendizaje, sean por ejemplo más prácticos.

El grado de preferencia mediano fue mayoritario en el estilo reflexivo y en el estilo teórico; sin embargo, la baja y alta preferencia distribuidas en la mayoría de participantes que usan los demás estilos denota la variedad de tendencias y que los estudiantes de medicina tienden a la práctica de un estilo o más (Tabla 2). En el estilo reflexivo, ponderante por sí mismo y junto al estilo teórico, al mejorar su preferencia mejora también el promedio ponderado, no siendo así en grupos con prevalencia de los demás estilos, en donde las calificaciones no mejoran su nivel al incrementar la preferencia por el estilo (Tabla 5).

El presente estudio coincide con los de Alzate y de Ángel y Alonso al tomar en cuenta las edades y estilo escogido. A pesar de la gran diferencia entre las edades mínima y máxima, en nuestro estudio la edad más frecuente fue 19 años, y el promedio no se alejó mucho de dicho resultado; el estilo reflexivo fue el de mayor tendencia.

Nuestro estudio también sugiere una diferencia en las tendencias a los estilos adoptados si se compara con otras carreras que estén fuera del área de ciencias de la salud. En el estudio de Cala, Riera y Jaramillo se observó que los estilos predominantes en alumnos de ingeniería fueron el pragmático y el activo; mientras que en el de Estrada y Alejandro, que investigo en alumnos de 
química los prevalentes fueron activo y teórico, cuando en estudios como el de Torres y Díaz, también dirigido a estudiantes de medicina humana, estos mismos estilos tuvieron el menor porcentaje de uso.

El estudio realizado por Torres y Díaz, no obstante, se realizó en alumnos cursando desde el primer al sexto año, a diferencia de nuestro estudio, aun así los resultados fueron similares en el orden de prevalencia de los diferentes estilos en ambos trabajos de investigación, ante esto surge el cuestionamiento de la necesidad de variar los estilos en estudiantes a lo largo de la carrera, o de mantener la ponderancia del estilo reflexivo. Al tener el médico un pensamiento crítico para resolver los problemas de su competencia, puede sugerirse como hipótesis para estudios posteriores que los mismos cursos orientan su perfil hacia el estilo reflexivo, sin embargo, en los últimos años de la carrera, los docentes sugieren que el estudiante aplique más los conocimientos a un ámbito práctico, por lo que sería razonable pensar la aplicación de métodos que orienten al uso del estilo pragmático-activo.

En este orden de sucesos y resultados en el caso de los estudiantes de medicina del primer año, la presente investigación demuestra que todos los estilos de aprendizaje son importantes y contribuyen a obtener un calificativo aprobatorio en los estudiantes, no existe correlación en este sentido puesto que realmente ningún estilo prevalece sobre otro, siendo que lo importante que se practique el estilo con el que más se sienta identificado el estudiante.

En este sentido para la presente investigación y para la realidad de estudio de los primeros años de estudio de los primeros años de medicina humana, el que los estudiantes practiquen un estilo de aprendizaje es importante, sin embargo al no estar correlacionado con la nota promedio no implica que cuanto más perfeccione el estilo mejora su nota promedio; lo que importa es que practique con naturalidad un estilo de aprendizaje en un nivel de mediana prevalencia.

Tabla 4: Correlación de los 4 estilos de aprendizaje con el rendimiento académico.

\begin{tabular}{|c|c|c|c|c|}
\hline & & & Estilo Teórico & $\begin{array}{c}\text { Nota } \\
\text { Promedio }\end{array}$ \\
\hline \multirow{7}{*}{$\begin{array}{l}\text { Rho de } \\
\text { Spearman }\end{array}$} & \multirow{3}{*}{ Estilo Teórico } & Coeficiente de correlación & 1,000 &,- 205 \\
\hline & & Sig. (bilateral) & . & 286 \\
\hline & & $\mathrm{N}$ & 29 & 29 \\
\hline & \multirow{3}{*}{ Nota Promedio } & Coeficiente de correlación &,- 205 & 1,000 \\
\hline & & Sig. (bilateral) &, 286 & . \\
\hline & & $\mathrm{N}$ & 29 & 29 \\
\hline & & & Estilo Activo & $\begin{array}{c}\text { Nota } \\
\text { Promedio }\end{array}$ \\
\hline \multirow{7}{*}{$\begin{array}{l}\text { Rho de } \\
\text { Spearman }\end{array}$} & \multirow{3}{*}{ Estilo Activo } & Coeficiente de correlación & 1,000 &,- 471 \\
\hline & & Sig. (bilateral) & . & 143 \\
\hline & & $\mathrm{N}$ & 11 & 11 \\
\hline & \multirow{3}{*}{ Nota Promedio } & Coeficiente de correlación &,- 471 & 1,000 \\
\hline & & Sig. (bilateral) &, 143 & . \\
\hline & & $\mathrm{N}$ & 11 & 11 \\
\hline & & & Estilo Reflexivo & $\begin{array}{c}\text { Nota } \\
\text { Promedio } \\
\end{array}$ \\
\hline \multirow{7}{*}{$\begin{array}{l}\text { Rho de } \\
\text { Spearman }\end{array}$} & \multirow{3}{*}{ Estilo Reflexivo } & Coeficiente de correlación & 1,000 &, 124 \\
\hline & & Sig. (bilateral) & . &, 137 \\
\hline & & $\mathrm{N}$ & 146 & 146 \\
\hline & \multirow{3}{*}{ Nota Promedio } & Coeficiente de correlación &, 124 & 1,000 \\
\hline & & Sig. (bilateral) &, 137 & . \\
\hline & & $\mathrm{N}$ & 146 & 146 \\
\hline & & & $\begin{array}{c}\text { Estilo } \\
\text { Pragmático } \\
\end{array}$ & $\begin{array}{c}\text { Nota } \\
\text { Promedio } \\
\end{array}$ \\
\hline \multirow{6}{*}{$\begin{array}{l}\text { Rho de } \\
\text { Spearman }\end{array}$} & \multirow{4}{*}{$\begin{array}{l}\text { Estilo } \\
\text { Pragmático }\end{array}$} & Coeficiente de correlación & 1,000 &,- 022 \\
\hline & & Sig. (bilateral) & . &, 924 \\
\hline & & $\mathrm{N}$ & 22 & 22 \\
\hline & & Coeficiente de correlación &,- 022 & 1,000 \\
\hline & \multirow[t]{2}{*}{ Nota Promedio } & Sig. (bilateral) &, 924 & . \\
\hline & & $\mathrm{N}$ & 22 & 22 \\
\hline
\end{tabular}

Fuente: Autores, Excel 2013. 
Tabla 5: Variación de los promedios ponderados según en grado de preferencia en los diferentes estilos.

\begin{tabular}{|c|c|c|c|c|}
\hline Estilo Reflexivo & $\begin{array}{c}\text { Baja } \\
\text { Preferencia }\end{array}$ & $\begin{array}{c}\text { Mediana } \\
\text { Preferencia }\end{array}$ & $\begin{array}{c}\text { Alta } \\
\text { Preferencia }\end{array}$ & Total \\
\hline $\mathrm{N}$ & 2 & 117 & 27 & 146 \\
\hline Media & 12.50 & 14.32 & 14.52 & 14.33 \\
\hline \multirow{2}{*}{$\begin{array}{l}\text { Desv. típ. } \\
\text { Error tip. de la } \\
\text { media }\end{array}$} & 1.90 & 0.92 & 0.75 & 0.93 \\
\hline & 1.34 & 0.09 & 0.14 & 0.08 \\
\hline Estilo Teórice & $\begin{array}{c}\text { Baja } \\
\text { Preferencia }\end{array}$ & $\begin{array}{c}\text { Mediana } \\
\text { Preferencia }\end{array}$ & $\begin{array}{c}\text { Alta } \\
\text { Preferencia }\end{array}$ & Total \\
\hline $\mathrm{N}$ & 3 & 15 & 11 & 29 \\
\hline Media & 14.61 & 14.19 & 13.94 & 14.14 \\
\hline \multirow{2}{*}{$\begin{array}{l}\text { Desv. típ. } \\
\text { Error típ. de la } \\
\text { media }\end{array}$} & 1.18 & 1.40 & 0.96 & 1.20 \\
\hline & 0.68 & 0.36 & 0.29 & 0.22 \\
\hline Estile Activo & $\begin{array}{c}\text { Baja } \\
\text { Preferencia }\end{array}$ & $\begin{array}{c}\text { Mediana } \\
\text { Preferencia }\end{array}$ & $\begin{array}{c}\text { Alta } \\
\text { Preferencia }\end{array}$ & Total \\
\hline $\mathrm{N}$ & 3 & 5 & 3 & 11 \\
\hline Media & 14.39 & 13.23 & 12.60 & 13.38 \\
\hline \multirow{2}{*}{$\begin{array}{l}\text { Desv. típ. } \\
\text { Error tip. de la } \\
\text { media }\end{array}$} & 1.18 & 0.97 & 1.56 & 1.28 \\
\hline & 0.68 & 0.43 & 0.90 & 0.39 \\
\hline Estilo Pragmático & $\begin{array}{c}\text { Baja } \\
\text { Preferencia }\end{array}$ & $\begin{array}{c}\text { Mediana } \\
\text { Preferencia }\end{array}$ & $\begin{array}{c}\text { Alta } \\
\text { Preferencia }\end{array}$ & Total \\
\hline $\mathrm{N}$ & 3 & 10 & 9 & 22 \\
\hline Media & 15.05 & 13.60 & 13.90 & 13.92 \\
\hline \multirow{2}{*}{$\begin{array}{l}\text { Desv. típ. } \\
\text { Error tip. de la } \\
\text { media }\end{array}$} & 1.05 & 0.49 & 1.16 & 0.98 \\
\hline & 0.61 & 0.16 & 0.39 & 0.21 \\
\hline$\frac{\text { Estilo Reflexivo y }}{\text { Teórico }}$ & $\begin{array}{c}\text { Baja } \\
\text { Prevalencia }\end{array}$ & $\begin{array}{c}\text { Mediana } \\
\text { Prevalencia }\end{array}$ & $\begin{array}{c}\text { Alta } \\
\text { Prevalencia }\end{array}$ & Total \\
\hline $\mathrm{N}$ & 7 & 7 & 1 & 15 \\
\hline Media & 14.13 & 14.54 & 14.39 & 14.34 \\
\hline Desv. típ. & 1.35 & 0.26 & & 0.92 \\
\hline $\begin{array}{l}\text { Error típ. de la } \\
\text { media }\end{array}$ & 0.51 & 0.10 & & 0.24 \\
\hline
\end{tabular}

Fuente: Autores, Excel 2013.

\section{CONCLUSIONES}

El estilo prevalente en los alumnos ingresantes de Medicina Humana de la Universidad Nacional Mayor de San Marcos es el Reflexivo, seguido por el Teórico y Pragmático, siendo el Activo el menos usado. La preferencia por el estilo reflexivo fue predominantemente moderada, mientras que para los demás estilos fue muy alta. El rendimiento académico de los estudiantes del primer año de medicina humana es indiferente al estilo de aprendizaje. Por tanto, lo que importa es que practique con naturalidad cualquier estilo de aprendizaje. Los datos presentados deben ser tomados en cuenta por la comunidad docente para la mejora del proceso enseñanza-aprendizaje. Resulta importante, como en su estudio lo hace Martínez, que el aprendizaje debería seguir un ciclo que empieza con una intención y estrategia para la búsqueda de datos (estilo activo), luego discutir, analizar sobre la información recopilada (estilo reflexivo), entonces armar un esquema concreto (estilo teórico) y al final practicar la teoría en situaciones reales (estilo pragmático).

\section{AUTOR DE CORRESPONDENCIA}

Dr. Hallder Mori Ramírez.

Punta Pejerrey 554.

Urbanización El Sol de La Molina

La Molina.

Teléfono: 998708015

E-mail: hmorir@unmsm.edu.pe, morihallder@gmail.com

\section{REFERENCIAS BIBLIOGRÁFICAS}

1. Da Cuña I, Gutierrez M, Barón F, Labajos M. Influencia del nivel educativo de los padres en el rendimiento académico, las estrategias de aprendizaje y los estilos de aprendizaje, desde la perspectiva de género. Revista de Estilos de Aprendizaje. 2014; 7 (3): 64-84.

2. Caballero M, Norambuena I, Gálvez J, Salamé A. Estilos de aprendizaje y rendimiento académico en estudiantes de Trabajo Social: un análisis entre México y Chile. Cuadernos de Trabajo Social- 
Universidad San Sebastián (Concepción, Chile). 2015; 14: 79-100.

3. Cala R, Riera M, Jaramillo $M$. Determinación de los estilos de aprendizaje de estudiantes de 1er curso de ing. Industrial y electrónica de la universidad técnica del norte. Ibarra. Ecuador. Revista de Estilos de Aprendizaje. 2014; 7 (14): 43-67.

4. Torres L, Diaz C. Estilos de aprendizaje y rendimiento académico en estudiantes de medicina humana de la Universidad San Martin de Porres filial norte. Julio-diciembre 2012. Rev Hisp Cienc Salud. 2016; 2(3): 232-241.

5. García A, Tamez C, Lozano A. Estilos de aprendizaje y rendimiento académico en alumnos de segundo grado de secundaria. Revista de Estilos de Aprendizaje. 2015; 8(15): 146-174.

6. Román L, Díaz L, Leyva E. Modificación de los estilos de aprendizaje durante la formación profesional, en estudiantes de enfermería. Revista de Estilos de Aprendizaje. 2015; 8 (16): 25-48.

7. Alvarado J, Montoya I, Rico A. Los estilos de aprendizaje y el rendimiento académico en matemáticas: aplicación del modelo de Honey y Mumford a una universidad colombiana. Revista de Estilos de Aprendizaje. 2017; 9 (18): 44-66.

8. Zambrano J, Arango L, Lezcano M. Estilos de aprendizaje, estrategias de aprendizaje y su relación con el uso de las TIC en estudiantes de educación secundaria. Revista de Estilos de Aprendizaje. 2018; 11 (21): 130-159.

9. Alzate, F. Estilos de aprendizaje en estudiantes colombianos de primer semestre de educación superior de la Universidad Abierta y a Distancia, UNAD. Trabajo de grado para optar por el título de Psicología, Facultad de Ciencias Sociales y Humanas, Universidad de Antioquia. 2014.

10. Martínez J, Carralero Y, Falcón Y, Guevara $\mathrm{R}$, Peña I. Influencia de los estilos de aprendizaje en el rendimiento académico en morfofisiología. Revista Electrónica Dr. Zoilo E. Marinello Vidaurreta. 2016; 41(7): 8 pp.

11. Estrada L, Alejandro A. Evaluación de estilos de aprendizaje en estudiantes de licenciatura en química de la Universidad Juárez Autónoma de Tabasco. Avances en Ciencias e Ingeniería. 2017; 8 (2): 47-52.

12. Díaz M, Ortega I. Caracterización de estilos de aprendizaje en estudiantes de ingeniería de jornadas diurnas y vespertinas. Revista
Enseñanza \& Teaching. 2017; 35 (2): $97-$ 108.

13. Escanero J, Soria S, Guerra M, Silva J. Comparación de los estilos de aprendizaje de los alumnos de medicina obtenidos con un nuevo cuestionario con los proporcionados por el cuestionario Honey-Alonso (CHAEA). Revista de la Fundación Educación Médica. 2016; 19 (1): 19-26.

14. Aravena C, Maureira F, Flores E, Lourido P, Véliz C. Independencia de los estilos de aprendizaje evaluados con el CHAEA-36 y el coeficiente intelectual en una muestra de estudiantes de secundaria de Chile. Revista Electrónica de Psicología Iztacala. 2017; 20(4): 1451-1462.

15. Vértiz B, Cardoso D, Bobadilla S. Estilos de aprendizaje. Caso estudiantes de psicología del centro universitario UAEM Temascaltepec. Revista Iberoamericana de las Ciencias Sociales y Humanísticas. 2015, 4 (17): 13 pp. 